\title{
Development of a suitable manufacturing process for production of a bioactive recombinant equine chorionic gonadotropin (reCG) in CHO-K1 cells
}

Javier Villarraza ${ }^{1}$, Sebastián Antuña ${ }^{2}$, Belén Tardivo ${ }^{2}$, María Celeste Rodríguez $^{1}$, Pablo

Mussio $^{1}$, Luciano Cattaneo ${ }^{3}$, Diego Fontana ${ }^{1}$, Natalia Ceaglio $^{4}$, and Claudio Prieto ${ }^{5}$

${ }^{1}$ UNL, CONICET, FBCB (School of Biochemistry and Biological Sciences), CBL

(Biotechnological Center of Litoral), Cell Culture Laboratory, Ciudad Universitaria, Ruta

Nacional 168 - Km 472.4 - C.C. 242 - (S3000ZAA) Santa Fe, Argentina

${ }^{2}$ Biotecnofe S.A., PTLC, Ruta 168 Pje El Pozo, (CP3000) Santa Fe, Argentina

${ }^{3}$ UNL, FCV, Cátedra de Teriogenología, Ruta Nacional 70- Km 472.4, Esperanza, Santa

Fe, Argentina

${ }^{4}$ Affiliation not available

${ }^{5}$ Universidad Nacional del Litoral Facultad de Bioquimica y Ciencias Biologicas

November 23, 2020

\begin{abstract}
Equine chorionic gonadotropin (eCG) is a heterodimeric glycoprotein hormone produced by pregnant mares that has been used to improve reproduction activity in different types of livestock. Several strategies to produce the hormone in a recombinant way have been reported; nevertheless, none approach has been able to produce a recombinant eCG (reCG) with significant in vivo bioactivity or in sufficient quantities for commercial purposes. For this reason, the only current product available on the market consists of partially purified preparations from serum of pregnant mares (PMSG). Herein, we describe a highly efficient process based on third-generation lentiviral vectors as delivery method for the production of reCG in suspension CHO-K1 cells, with productivities above 20 IU.106 cell-1.d-1 and $70 \%$ purification yields after one purification step. Importantly, reCG not only demonstrated biological activity in bovine cattle but also this bioactivity appeared to be higher than PMSG, since 140 IU of reCG were needed to exert the same biologic effect in an ovulation synchronization protocol compared to 400 IU of PMSG. The results obtained show that the developed strategy represents an attractive option to produce reCG and constitutes an auspicious alternative for the replacement of animals as a source of PMSG.
\end{abstract}

Development of a suitable manufacturing process for production of a bioactive recombinant equine chorionic gonadotropin (reCG) in CHO-K1 cells

Carlos J Villarraza ${ }^{1}$, Sebastián Antuña ${ }^{5}$, María B Tardivo ${ }^{5}$, María C Rodríguez ${ }^{1}$, Pablo Mussio ${ }^{2}$, Luciano Cattaneo $^{3}$, Diego Fontana ${ }^{1,4,5}$, Natalia A Ceaglio ${ }^{1}$, Claudio C Prieto ${ }^{2,4,5}$

${ }^{1}$ UNL, CONICET, FBCB (School of Biochemistry and Biological Sciences), CBL (Biotechnological Center of Litoral), Cell Culture Laboratory, Ciudad Universitaria, Ruta Nacional 168 - Km 472.4 - C.C. 242 (S3000ZAA) Santa Fe, Argentina

${ }^{2}$ UNL, FBCB (School of Biochemistry and Biological Sciences), CBL (Biotechnological Center of Litoral), Biotechnological Development Laboratory, Ciudad Universitaria, Ruta Nacional 168 - Km 472.4 - C.C. 242 - (S3000ZAA) Santa Fe, Argentina 
${ }^{3}$ UNL, FCV, Cátedra de Teriogenología, Ruta Nacional 70- Km 472.4, Esperanza, Santa Fe, Argentina.

${ }^{4}$ Cellargen Biotech S.R.L., Antonia Godoy 6369 (S3000ZAA) Santa Fe, Argentina

${ }^{5}$ Biotecnofe S.A., PTLC, Ruta 168 Pje El Pozo, (CP3000) Santa Fe, Argentina

*Corresponding author: Claudio Prieto. Mail: cprieto@fbcb.unl.edu.ar

Keywords: reCG, PMSG, Glycoprotein hormones, Lentiviral Particles (LP) and Suspension CHO-K1

Abbreviations: CHO, Chinese Hamster Ovary; FSH, follicle-stimulating hormone; HEK, Human Embryonic Kidney; IVD, intravaginal device; LH, luteinizing hormone; LP, lentiviral particles; OR, ovulation rate; OT, ovulation time; PMSG, Pregnant Mare Serum Gonadotropin; POF, preovulatory follicle, reCG, recombinant equine chorionic gonadotropin; SFM , serum free medium; TSH, thyroid-stimulating hormone

\section{ABSTRACT}

Equine chorionic gonadotropin (eCG) is a heterodimeric glycoprotein hormone produced by pregnant mares that has been used to improve reproduction activity in different types of livestock. Several strategies to produce the hormone in a recombinant way have been reported; nevertheless, none approach has been able to produce a recombinant eCG (reCG) with significant in vivo bioactivity or in sufficient quantities for commercial purposes. For this reason, the only current product available on the market consists of partially purified preparations from serum of pregnant mares (PMSG). Herein, we describe a highly efficient process based on third-generation lentiviral vectors as delivery method for the production of reCG in suspension CHO-K1 cells, with productivities above $20 \mathrm{IU}^{-1} 10^{6} \mathrm{cell}^{-1} \cdot \mathrm{d}^{-1}$ and $70 \%$ purification yields after one purification step. Importantly, reCG not only demonstrated biological activity in bovine cattle but also this bioactivity appeared to be higher than PMSG, since $140 \mathrm{IU}$ of reCG were needed to exert the same biologic effect in an ovulation synchronization protocol compared to $400 \mathrm{IU}$ of PMSG. The results obtained show that the developed strategy represents an attractive option to produce reCG and constitutes an auspicious alternative for the replacement of animals as a source of PMSG.

\section{INTRODUCTION}

Equine chorionic gonadotropin (eCG) belongs to the glycoprotein hormone family together with folliclestimulating hormone (FSH), luteinizing hormone (LH) and thyroid stimulating hormone (TSH). ${ }^{[1]}$ The eCG was first described in pregnant mares' serum, having this serum the ability to stimulate gonadal development in rats and mice. ${ }^{[2]}$ For this reason, eCG was originally called Pregnant Mare Serum Gonadotropin (PMSG).

Like other members of the glycoprotein hormone family, eCG is a heterodimeric protein composed by two different non-covalently linked subunits, named $\alpha$ and $\beta$. Within the same species, the $\alpha$ subunit is common to all members of the family and it is encoded by a single gene, while different genes encode the $\beta$ subunits, which confer specificity to heterodimers. ${ }^{[3]} \alpha$ subunit of $\mathrm{eCG}(\mathrm{eCG} \alpha)$ is composed by 96 amino acids and it exhibits two sites for the attachment of N-linked glycans, located at Asn56 and Asn82. ${ }^{[4-7]} \beta$ subunit (eCG $\beta$ ) is composed by 149 amino acids and contains only one N-linked glycosylation site at Asn13. In addition, a distinctive feature of eCG $\beta$ is the presence of a carboxyl terminal peptide (CTP) composed by 28 amino acids (122-149) that contains 12 O-glycosylation sites in Ser o Thr residues. ${ }^{[8-10]}$

eCG has two distinctive characteristics compared to the other glycoprotein hormones. On the one hand, in species other than equine, eCG shows high FSH- and LH-like activity and has a high affinity for the receptors for these hormones. ${ }^{[11]}$ On the other hand, it exhibits a high content of carbohydrates, which constitute $45 \%$ of its total molecular weight. This last property determines the long circulating half-life of eCG. ${ }^{[12-15]}$ Due to both characteristics, eCG is used in veterinary to control reproductive activity in different types of livestock, including cattle, sheep, goats and pigs. ${ }^{[16]}$

Currently, the products available in the market are partially purified eCG preparations from blood of pregnant mares (PMSG), comprising many disadvantages. On the one hand, they show batch-to-batch variations, since the glycosylation profile (which considerably influences the half-life and, consequently, the efficacy of 
the hormone) varies between animals and between sera at different stages of gestation. On the other hand, PMSG may also contain contaminants with potential health risks. This goes against the current trend of regulatory entities to obtain safer veterinary products, free of viruses, prions and other contaminating proteins. Last but not least, the practice to which the animal is subjected compromises animal welfare, and it is completely questionable from the bioethical point of view: it is a bloody process which may generate significant anemias and, in some cases, ends with the animal's life.

For this reason, the development of a recombinant eCG (reCG) as a substitute of PMSG is imperative. This approach not only allows a better control of the production source, replacing the use of animals, but also ensures greater batch-to-batch consistency and reproducibility, and eliminates any bioethical concern. ${ }^{[17]}$

The importance of a correct glycosylation profile for eCG to exhibitin vivo bioactivity has been described. ${ }^{[18-21]}$ Chinese Hamster Ovary(CHO-K1) cell line represents an attractive host for the expression of recombinant eCG that exhibits in vivo FSH/LH bioactivity. CHO-K1 cells express a wide range of glycosyltransferases, which are capable of adding complex sialylated N-glycans to the polypeptides. ${ }^{[22]}$ In this sense, the expression of recombinant eCG from transfected CHO-K1 cells that exhibits in vivo LH-like activity in horse breeding stallion and cyclic mares has been reported. ${ }^{[23,24]}$ Nevertheless, the authors did not report LH/FSH-like activity in species other than the horse.

Here we describe the production, purification and characterization of a biologically active recombinant equine chorionic gonadotropin expressed in CHO-K1 cells cultured in serum free medium (SFM). To our knowledge, this is the first report of a recombinant eCG demonstrating bioactivity in target species, specifically, in bovine cattle. Furthermore, this is the first time that lentiviral vector technology has been applied to deliver reCG transgenes in manufacturing cell lines for veterinary use.

\section{MATERIAL AND METHODS}

2. $\mathbf{e C G}$

3. $P M S G$

Commercial preparations of PMSG were purchased from regional veterinary drugstores.

Highly pure reCG

A batch of highly pure reCG was prepared by RP-HPLC. The eluted protein with high purity (>99\%) was prepared with the aim of being used in an immunization protocol and a competitive ELISA, as described below (see section 2.7 ). Concentration of this batch of reCG was estimated by measurement of absorbance at $280 \mathrm{~nm}$. A mass extinction coefficient of $0.43 \mathrm{l} \cdot \mathrm{g}^{-1} \cdot \mathrm{cm}^{-1}$ (estimated using Vector NTI(r) program) was used to convert absorbance units into mass units.

\section{Antibodies}

The polyclonal anti-reCG antibodies (pAb) were obtained in our lab by immunization of a rabbit with highly pure reCG using a standard protocol. ${ }^{[25]}$ Commercial peroxidase-conjugated goat anti-rabbit immunoglobulins (DAKO, Denmark) were employed.

\section{Plasmid construction and lentivirus production}

The coding sequence of each reCG subunit was cloned in the self-inactivating (SIN) lentiviral vector (pEF$1 \alpha,{ }^{[26]}$ which contains the sequence of the human elongation factor 1 alpha driving the expression of the transgene. Two transfer vectors were generated: $\mathrm{pEF}-1 \alpha-\mathrm{reCG} \alpha$ and $\mathrm{pEF}-1 \alpha-\mathrm{reCG} \beta$.

For the production of lentiviral particles (LP), adherent HEK293T/17 cells $\left(8 \times 10^{5}\right.$ cells per well) were cotransfected with four plasmids: the packaging construct (pMDLg/pRRE), the VSV-G-expressing construct (pMD.G), the Rev-expressing construct (pRSV-Rev), ${ }^{[27,28]}$ and the respective transfer vector pEF- $1 \alpha$-reCG $\alpha$ or pEF-1 $\alpha$-reCG $\beta$. Polyethyleneimine (PEI) was used as a DNA carrier. The supernatants containing LP eCG $\alpha$ or LP eCG $\beta$ were harvested after $72 \mathrm{~h}$ and clarified by low-speed centrifugation. After that, the LPs were concentrated by centrifugation at $65.000 \mathrm{x}$ g, resuspended in SFM and frozen at $-70{ }^{\circ} \mathrm{C}$ prior to use. 
The titer was calculated using a HIV-1 p24 ELISA kit (QuickTiter ${ }^{\mathrm{TM}}$ Lentivirus Titer Kit, Cell Biolabs Inc., USA) following manufacturer's recommendations.

\section{Cell culture, cell lines generation and clonal isolation}

Suspension adapted-CHO-K1 cells (sCHO-K1) were cultured in serum free-medium at $37{ }^{\circ} \mathrm{C}$ with $5 \% \mathrm{CO}_{2}$. The transduction event was performed by incubating $5 \times 10^{3}$ cells with both LP eCG $\alpha$ and LP eCG $\beta$ in 96-well plates in a final volume of $100 \mu \mathrm{l}$ of SFM. A multiplicity of infection (MOI) of $30 \mathrm{LP}$ per cell for each LP was used. After $72 \mathrm{~h}$ of incubation, supernatant medium was replaced with fresh SFM and transduced cells were cultured for another round of transduction as outlined above.

The recombinant cell lines were subjected to increasing levels of puromycin in order to get rid of the remaining untransduced cells. After selective pressure, the supernatant of each cell line was analyzed by western blot to screen the level of recombinant protein expression.

The selected cell line was cloned in 96-well plates using the limiting dilution method. ${ }^{[29,30]}$ After 14 days, single-cell clones were selected, and the amount of protein expressed by each clone was analyzed by dot-blot. The highest expressing clones were isolated and amplified, and the supernatant of selected clones and the parental cell line were screened by western blot.

\section{Determination of reCG productivity}

To determine reCG productivity of each cell line and clone, cells were seeded in CellSTARß CellReactor ${ }^{\text {TM }}$ tubes at $1 \times 10^{6}$ cell.ml $^{-1}$. After $48 \mathrm{~h}$ cell density was estimated by cell counting in hemocytometer and reCG concentration in the supernatant was determined using a competitive ELISA, as described below (see sections 2.7 ).

\section{Scale-up and reCG production}

reCG producing CHO-K1 cells were grown in Erlenmeyer flasks (Corning, USA) in a shaker incubator (Certomat@CTplus, Sartorius, Germany) at $37{ }^{\circ} \mathrm{C}$ with $5 \% \mathrm{CO}_{2}$ and a shaking speed of $140 \mathrm{rpm}$. Thereafter, cells were transferred to a one-liter bioreactor (BIOSTAT\& Qplus, Sartorius) and cultured in perfusion mode. The culture parameters were controlled online, maintaining a $\mathrm{pO}_{2}$ of $30 \%$ air-saturation, a $\mathrm{pH}$ of 7.1 and an agitation rate of $140 \mathrm{rpm}$. Temperature was varied between $37 \mathrm{oC}$ and $31 \mathrm{oC}$. Samples were collected daily to determine cell density, cell viability, glucose and lactate levels and reCG concentration.

\section{Cell counting and viability determination}

Cell were counted in a Neubauer chamber, estimating the proportion of dead cells by the trypan blue exclusion method. ${ }^{[31]}$

\section{Metabolite Analysis}

Glucose and lactate determinations were performed with a reflectometer (RQflex 2, Merck, USA), using Reflectoquant(r) test strips containing specific immobilized enzymes for glucose and lactate. Samples were incubated with the test strips and analyte concentration was estimated reflectometrically. Measuring ranges were 1-100 $\mathrm{mg} \mathrm{l}^{-1}$ and 3-60 $\mathrm{mg} \mathrm{l}^{-1}$ for glucose and lactate, respectively.

\section{reCG quantification}

reCG quantification was performed using a competitive ELISA developed in our laboratory. Briefly, the assay involved competition between the solid phase-immobilized antigen (reCG) with the same antigen (reference reCG or unknown sample) in solution for binding to the pAb anti-reCG. Subsequently, peroxidase-conjugated secondary antibodies were added to detect the remaining solid phase bound complexes. Finally, the assay was revealed by incubation with o-phenylenediamine (Sigma-Aldrich) solution followed by measurement of absorbance at $492 \mathrm{~nm}$. Highly pure reCG was used as immobilized antigen and partially purified reCG with knownin vivo biopotency was used as reference (IU.ml-1).

\section{reCG Purification}


Supernatants containing reCG were clarified by filtration with $0.45 \mu \mathrm{m}$ membrane filters (Sartobran P, Sartorius). Clarified supernatants were loaded onto a CaptoBlue-Sepharose FF resin packed on an XK 16/20 column (GE Healthcare) and equilibrated in $20 \mathrm{mM}$ Tris-HCl pH 7 buffer, connected to an ÄKTA explorer 100 Air (GE Healthcare). The column was washed with equilibration buffer and then reCG was eluted with NET elution buffer ( $\mathrm{NaCl} /$ ethanol/Tis). The eluted protein was diafiltered and concentrated against phosphate-buffered saline (PBS) using Microsep@ Advanced Centrifugal Devices (PALL Corporation, USA).

\section{Physicochemical Characterization}

\section{$S D S-P A G E$}

Electrophoresis under non-reducing conditions was performed according to Laemmli ${ }^{[32]}$ using the Mini Protean 3 system (Bio-Rad, EE.UU.). Resolving and stacking gels were prepared at $12 \%$ and $5 \%$ (w/v) polyacrylamide, respectively. Separation was performed at $200 \mathrm{~V}$ for $70 \mathrm{~min}$. Gels were stained with Coomassie R-2500 and destained with a solution containing $15 \%$ (v/v) methanol and $10 \%$ (v/v) acetic acid. For western blot, proteins were transferred to a polyvinylidene difluoride (PVDF) membrane (Bio-Rad) according to Hames ${ }^{[33]}$ during $1 \mathrm{~h}$ at $180 \mathrm{~mA}$ in $25 \mathrm{mM}$ Tris, $0.2 \mathrm{M}$ glycine and $20 \%(\mathrm{v} / \mathrm{v}$ ) methanol.

Isoelectric Focusing (IEF)

Separation of reCG isoforms was performed by isoelectric focusing using a 1-mm thick $8 \%$ (w/v) polyacrylamide gel containing $8 \mathrm{M}$ urea. The $\mathrm{pH}$ range was established using $75 \%$ (w/v) 3-5 ampholytes and $25 \%$ (w/v) 5-7 ampholytes (GE Healthcare). Ampholytes were pre-focused for $30 \mathrm{~min}$ at $30 \mathrm{~W}, 250 \mathrm{~V}$ and 50 $\mathrm{mA}$ in order to generate the $\mathrm{pH}$ gradient. Then, $20 \mu \mathrm{l}$ of the samples were applied over a sample application strip placed at $1 \mathrm{~cm}$ from cathode. Electrophoresis was performed for $90 \mathrm{~min}$ under the same conditions as described in pre-focusing. After electrophoresis, proteins were transferred onto PVDF membrane using the Multiphor II Nova Blot (GE Healthcare) system for $1 \mathrm{~h}$ at $10 \mathrm{~W}, 2000 \mathrm{~V}$ and $150 \mathrm{~mA}$.

Dot-blot

To compare the levels of reCG produced by the different clones during the cloning procedure, a dot-blot assay was used. For this, the supernatants from each clone were immobilized on a nitrocellulose membrane (BioRad) using the Bio-Dot@ Microfiltration Apparatus (Bio-Rad) following the manufacturer's instructions.

\section{Immunochemical detection}

After transference or immobilization, membranes were blocked with Tris-buffered saline (TBS) containing $0.05 \%(\mathrm{v} / \mathrm{v}$ ) Tween 20 (TBS-T) and 5\% non-fat milk. Proteins were detected with appropriately diluted rabbit polyclonal anti-reCG antibodies in TBS-T containing $0.5 \%$ non-fat milk. Peroxidase-conjugated goat anti-rabbit Ig was used as a secondary antibody. Protein bands were revealed using a chemiluminescent reagent (Pierce ${ }^{\mathrm{TM}}$ ECL Western Blotting substrate, Thermo Fisher Scientific, USA).

\section{In vivo bioassays}

\section{Potency bioassay}

The in vivo bioactivity of purified reCG was evaluated in female rats following the eCG assay of Cole and Erway ${ }^{[34]}$ recommended by European Pharmacopoeia using the WHO International Standard for serum equine gonadotropin (NIBSC 62/001). Three doses of each hormone were tested by s.c. injection of five animals. The assay was conducted at the Centro de Medicina Comparada (CMC, ICIVET, Esperanza, Santa Fe, Argentina).

\section{Target species bioassay}

Sixty-three crossbred Bos taurus x Bos indicus anestrus suckling cows after 60-90 days from partum were included in the study. The study was carried out on a farm placed in the middle west of Santa Fe province under subtropical conditions. Cows were kept in natural pastures with none supplementation around the year. Body condition score (BCS) was assessed using a 1-5 scale (1= emaciated, $5=$ obese). ${ }^{[5]}$ Anestrus condition 
was evaluated by two ultrasound exams: one at day 0 of the protocol and the second at intravaginal device (IVD) withdrawal (day 7). In both cases, anestrus was confirmed by the absence of corpus luteum and $<10 \mathrm{~mm}$ follicles. Study design and protocol are shown in Fig. 1 . Basically, on day 0 cows received a $0.75 \mathrm{~g}$ progesterone (P4) IVD and a $2 \mathrm{mg}$ injection of estradiol benzoate (EB). On day 7 , the device was removed and $0.150 \mathrm{mg}$ of D-Cloprostenol (PGF-2 $\alpha$ ) and $1 \mathrm{mg}$ of estradiol cypionate (EC) were administered intramuscularly. At that day, cows were blocked according to BCS and size of the dominant follicle (DF), and randomly distributed in 3 groups: 1) control group $(n=22)$, cows were not supplemented with eCG; 2) reCG group $(n=20)$, cows were given a 140 IU injection of reCG; and 3) PMSG group ( $n=21)$, cows were injected with $400 \mathrm{IU}$ of PMSG (Novormon), according to the manufacturer. From day 9 (48 h after IVD withdrawal) until day 12 cows were examined daily by ultrasound to determine preovulatory follicle (POF) size and ovulation. One last ultrasound examination was performed on day 23 (mid diestrus) to measure corpus luteum (CL) size. In all cases, DF diameter was calculated as the average of the vertical and horizontal diameters. The same criterion was applied for CL size determination. For those CL with cavity, average diameter of the cavity was calculated and extracted from total CL diameter.

\section{Statistical analysis}

Statistical analyses of potency bioassay results were performed using ANOVA test, employing Statgraphics Centurion XVI. Differences between treatments were considered significant when $p<0.05$. To determine which means were significantly different from others (multiple comparison) a Fisher's minimum significant difference (LSD) method was applied.

In the case of target species bioassay, a generalized lineal model (GLM) with binomial distribution and logit link function was applied for the dichotomous variable "ovulation rate (OR)". For the continuous variables "day 7 DF diameter (DFDd7)", "day 9 POF diameter (POFDd9)" and "CL diameter (CLD)", a GLM with normal distribution and lineal relationship was performed. For the variable ovulation time (OT, IVD removal to ovulation interval), a Kaplan-Meier survival test was applied. In these cases, the statistical package SPSS was used.

\section{RESULTS}

\section{Cell culture, cell lines generation and clonal isolation}

Two events of co-transduction of sCHO-K1 cells were performed with both LPs coding for each eCG subunit, generating two cell lines named reCG Td1 and reCG Td2. The aim of this serial transduction process was to increase the copy number of the transgenes and, consequently, the level of protein expression. To eliminate the remaining untransduced cells, the recombinant cell lines were treated with puromycin. As expected, production of reCG increased after the second co-transduction event. The productivity of Td2 cell line was almost four times higher compared with that of Td1 cell line $\left(5.6 \pm 0.2 \mathrm{IU}_{10} 10^{6} \mathrm{cell}^{-1} . \mathrm{d}^{-1}\right.$ for Td1 versus $22 \pm$ 1 IU. $10^{6}$ cell $^{-1} \cdot \mathrm{d}^{-1}$ for Td2, $\left.\mathrm{p}<0.05\right)$.

Considering these results, the reCG Td2 cell line was selected to be cloned by limit dilution method. A total of 400 clones were analyzed by dot blot in three pre-selection rounds. Clones selected after each round were subcultured and re-evaluated in a new round of selection until five of the highest producing clones could be identified. Western blot analysis of supernatants of the five selected clones revealed that clones P5C3 and P5D9 exhibited the highest reCG production level (data not shown). Despite the productivity of both clones was slightly lower regarding the one of the parental cell line $\left(20.6+-0.4 \mathrm{IU}^{-1} 0^{6}\right.$ cell $^{-1} . \mathrm{d}^{-1}$ for P5C3 and 17.5 +- 0.7 IU. $10^{6}$ cell $^{-1} . \mathrm{d}^{-1}$ for P5D9), P5C3 clone was selected for scaling up.

\section{Scale-up. reCG production and purification}

$\mathrm{P} 5 \mathrm{C} 3$ clone was cultured in SFM in a one-liter bioreactor in perfusion mode for 19 days, reaching a maximum cell density of $2.9 \times 10^{7}$ cells.ml ${ }^{-1}$ (Fig. 2 ). Cell viability was above $95 \%$ during the complete cultivation process. The perfusion rate varied between 0.2 and 1.5 reactor volumes per day, as the process demanded. Importantly, lactate concentration stayed below $15 \mathrm{mM}$, evading the toxic effects that this metabolite causes at concentrations above $20-30 \mathrm{mM}$, including the alteration of the $\mathrm{pH}$ and osmolarity of the medium. ${ }^{[36]}$ 
As the cell concentration increased, the temperature of the culture was reduced in order to decrease the

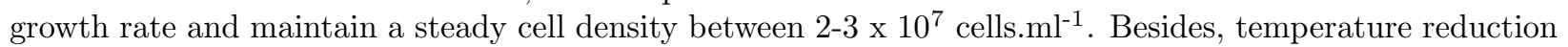
represents a typical proliferation control strategy to improve cell productivity. ${ }^{[37]}$ The specific growth rate calculated during exponential phase was $0.013 \mathrm{~h}^{-1}$.

Clarified supernatant containing 100-258 IU.ml ${ }^{-1}$ of reCG was loaded onto a previously equilibrated CaptoBlue-Sepharose FF resin. After washing the column, reCG was eluted with a yield around $70 \%$ (Fig. 3 ).

\section{Physicochemical characterization}

In order to characterize the apparent molecular mass of the recombinant hormone, partially purified reCG was electrophoresed under three different conditions: A, unheated and non-reducing conditions; B, heated and non-reducing conditions; C, heated and reducing conditions. Supernatants of untransduced CHO-K1 cells (sCHOwt) and PMSG were assayed under the same conditions as controls. Blots were then probed with anti-reCG serum (Fig. 4 ). On the one hand, the apparent molecular mass of the reCG heterodimer was about $46 \mathrm{kDa}$ with bands ranging from $40-52 \mathrm{kDa}$ (Fig. 4A , lane 2). Traces of free $\alpha$ subunit were also detected, with an apparent molecular mass ranging from 21 to $28 \mathrm{kDa}$. When samples were heated, the reCG dimer was dissociated in each of its subunits (Fig. 4B , lane 2). This was evidenced by the disappearance of the band around $46 \mathrm{kDa}$, the appearance of a new band corresponding to $\beta$ subunit of around $29-37 \mathrm{kDa}$, and the consequent increase in the intensity of the band corresponding to the $\alpha$ subunit. The identity of these bands was confirmed by the absence of any bands in the supernatant of sCHOwt (Fig. $\mathbf{4 A}$ and $4 \mathbf{B}$ , lane 3). On the other hand, PMSG exhibited a very different electrophoretic profile, comprising a wide range of bands with higher molecular masses ranging from 50 to $72 \mathrm{kDa}$, and some bands above $100 \mathrm{kDa}$ (Fig. 4A, lane 1). When samples were subjected to reducing conditions, the antibody was unable to detect neither reCG nor PMSG (Fig. 4C , lanes 1 and 2), suggesting that the antibody can only bind the hormone when its tertiary structure is stabilized by the presence of the disulfide bridges in each subunit.

To characterize reCG and PMSG isoform profile, IEF assays followed by western blot were performed (Fig. 5 ). PMSG exhibited a greater number of isoforms than reCG, distributed practically throughout the $\mathrm{pH}$ range, with prevalence of more acidic isoforms, probably due to a higher content of sialic acid residues. Partially purified reCG showed fewer isoforms located in the less acidic range of the gel, i.e. with a higher $\mathrm{pI}$ than PMSG. Similar results were obtained with supernatants containing reCG, indicating that all glycoforms were recovered after the pseudoaffinity purification process (data not shown).

\section{In vivo bioassay: Potency bioassay}

The biopotency of partially purified reCG was evaluated using the assay recommended by the European Pharmacopoeia. The estimated biopotency for reCG was $7200 \pm 100 \mathrm{IU} \cdot \mathrm{ml}^{-1}$, demonstrating that the recombinant hormone produced under these conditions is biologically active. Furthermore, the specific biological activity was $7500+-200 \mathrm{IU}^{\mathrm{m}} \mathrm{mg}^{-1}$, a value considerably higher than the minimum required by the European Pharmacopoeia 8.0 (1000 IU. $\left.\mathrm{mg}^{-1}\right)$.

\section{Target species bioassay}

The negative energy balance after partum, which is highly influenced by low body condition at calving, in addition to suckling in beef cattle ${ }^{[38,39]}$ or to high milk production in dairy cattle, ${ }^{[40,41]}$ are the main driving factors of anestrus in cows. Under these conditions, LH pulsatility is reduced, compromising the final stages of follicular growth, maturation and ovulation. ${ }^{[42]}$ Nunez-Olivera et al. ${ }^{[39]}$ have demonstrated that the administration of $400 \mathrm{IU}$ of eCG from pregnant mare serum (PMSG) at IVD removal in an ovulation synchronization protocol enhanced ovulation rate and increased the size of the corpus luteum and the progesterone concentration in serum.

Thus, the effects of the administration of reCG on follicular growth, ovulation rate, ovulation time and size of corpus luteum at the end of an ovulation synchronization protocol were studied in comparison with PMSG (Table 1 ). 
No significant differences were found between groups in BCS, DFDd7 and DFDd9. However, ovulation rate was significantly higher for both eCG-treated cows in comparison to control cows (reCG $=80 \%, 16 / 20$; $\mathrm{PMSG}=71 \%, 16 / 21 ;$ control $=41 \%, 9 / 22)$. Significant differences were also found in OT, being the OT earlier in the control and reCG groups compared to PMSG-treated cows. Finally, corpus luteum diameter was similar in all groups.

\section{DISCUSSION}

Equine chorionic gonadotropin is an important hormone used in veterinary to control reproductive activity in different types of livestock. ${ }^{[16]}$ Currently, the only commercial product available for this purpose consists of a partially purified preparation of plasma from pregnant mares (PMSG), which not only constitutes a health risk due to the contaminants present in the plasma, but also raises serious bioethical questions. Several attempts to produce recombinant eCG have been reported in different hosts likePichia pastoris and insect cells (Sf9 and Mimic ${ }^{\mathrm{TM}}$ ). Nevertheless, in all cases, the hormone exhibited no significant in vivo bioactivity in rat models, which the authors attributed to its extremely short circulatory half-life caused by the absence of terminal sialic acid in the oligosaccharide chains. ${ }^{[19-21]}$ These results reveal the importance of a correct glycosylation profile for eCG to exhibitin vivo bioactivity. In particular, reCG obtained from CHO-K1 cells demonstrated in vivo bioactivity in equine models, ${ }^{[23,24]}$ but activity in species other than the horse have not been reported. Despite this, commercial versions of reCG are not yet available on the market. This result evidences a problem to be solved, which consists in the fact that large amounts of recombinant eCG cannot be obtained in an "efficient way", or with a glycosylation profile similar to that of natural eCG, which guarantees it's in vivo biological activity. ${ }^{[43]}$ Under these circumstances, we herein report the production of a target species-bioactive reCG in suspension CHO-K1 cells cultured in serum-free medium, using third-generation lentiviral vector system as a suitable expression platform.

Indeed, the use of lentivirus as a gene delivery system allowed the development of highly producer cell lines and clones, with productivities above $20 \mathrm{IU} \cdot 10^{6} \mathrm{cell}^{-1} \cdot \mathrm{d}^{-1}$. P5C3 clone cultured in a one-liter bioreactor produced almost $1.8 \times 10^{5} \mathrm{IU}$ per day. Considering that one dose of reCG corresponds to $140 \mathrm{IU}$, the process here developed could produce up to 1300 doses per day in a laboratory scale. If these results are extrapolated to a production scale of, e.g. 50-liter bioreactor, the number of doses obtained could reach up to $6.5 \times 10^{4}$ doses per day. Therefore, in a production scale we could produce in a 25-30 days bioprocess the same number of doses than 600-700 pregnant mares in 200 days. By this, we can reduce the time and cost of the overall eCG production process replacing the use of animals as source of this hormone. Results reported herein demonstrate, once more, the potential of lentiviral vectors to deliver transgenes in manufacturing cell lines. Moreover, after one purification step, the hormone was recovered with a $70 \%$ yield. This value could be considered adequate to produce a recombinant hormone for veterinary use, with a higher purity than the PMSG available on the market for the same purpose.

Regarding glycosylation profile, reCG presented a lower number of glycoforms than PMSG, which would indicate a greater homogeneity of the recombinant hormone compared to the natural variant. The PMSG also showed a predominance of more acidic isoforms, which in turn exhibited a lower $\mathrm{pI}$ than the more acidic isoforms present in the reCG. These results suggest that the recombinant hormone exhibits a lower content of sialic acid compared to PMSG. In accordance with what was found in the SDS-PAGE and western blot assays, this would be a consequence of the lower degree of glycosylation of reCG, either in terms of the occupation degree of the potential $\mathrm{N}$ - and O-glycosylation sites, or in terms of the complexity of the glycan structures attached to these sites. Our findings agree with those previously reported by Jablonka-Shariff et al. ${ }^{[23]}$, who affirm that the product obtained by culturing recombinant CHO-K1 cells does not reach the degree of glycosylation of PMSG.

Purified reCG demonstrated in vivo biological activity in female rats with a specific biological activity around $7500 \mathrm{IU}_{\mathrm{mg}}{ }^{-1}$. Moreover, despite the different glycosylation profile of reCG with respect to PMSG, the recombinant hormone produced in this study demonstrated an in vivo biological activity in cattle, being the first time that this type of activity is reported in bovines. Indeed, we could demonstrate that the application of $140 \mathrm{IU}$ of reCG significantly increased the ovulation rate compared with those cows that 
were not supplemented with this gonadotropin, and that was comparable to the effect of 400 IU of PMSG. In previous studies, Nunez-Olivera et al. ${ }^{[39]}$ mentioned that POFD on day 9 tended to be larger in cows supplemented with PMSG ( $\mathrm{p}=0.07)$. In our case, we found a numeric difference, but it was not significant $(\mathrm{p}=0.26)$. Moreover, in the study performed by Nunez-Olivera et al. ${ }^{[39]}$, they could demonstrate that those animals receiving PMSG showed larger CL and higher P4 serum concentration than the control group. In the present study, we did not find a significant difference in CL size between treatments $(p=0.157)$. This could be related to the fact that we performed only one US exam to determine CLD, whereas Nunez-Olivera et al. ${ }^{[39]}$ performed daily US exams for several days during the diestrus. On another aspect, it could be observed that cows from the control and reCG groups ovulated significantly earlier that cows from the PMSG group $(\mathrm{p}=0.049)$.

To our knowledge, this is the first published study that demonstrates the effects of a recombinant eCG on follicular growth, ovulation and CL formation in anestrus cows. Furthermore, bioactivity of the recombinant hormone in cattle seems to be higher than PMSG, since a reduced number of IU of reCG are needed to exert the same biologic effect in an ovulation synchronization protocol compared to PMSG.

\section{CONCLUSION}

In this study, we report the development of a highly efficient process to produce recombinant equine chorionic gonadotropin (reCG) in CHO-K1 cells using lentiviral vector systems as a delivery method. The results obtained show that the developed strategy represents an attractive option to produce reCG and constitutes an auspicious alternative for the replacement of animals as a source of PMSG.

\section{ACKNOWLEDGEMENTS}

This work was supported by the Consejo Nacional de Investigaciones Cientificas y Tecnicas (CONICET), Argentina; the Ministerio de Ciencia, Tecnologia e Innovacion Productiva de Santa Fe (MinCTIP) and the Universidad Nacional del Litoral (UNL), Argentina.

\section{CONFLICT OF INTEREST}

The authors declare no commercial or financial conflict of interest

\section{REFERENCES}

[1] B.D. Murphy, S.D. Martinuk, Endocr. Rev. 1991 ,12, 27.

[2] H.H. Cole, G.H. Hart, Am. J. Physiol. Content1930 , 93 , 57.

[3] F. Stewart, W.R. Allen, J. Reprod. Fertil. 1979 ,27, 431.

[4] D.N. Ward, W.T. Moore, B.D. Burleigh, J. Protein Chem.1982, 1 , 263.

[5] D.N. Ward, G.R. Bousfield, in Glycoprotein Horm. Struct. Synth. Biol. Funct. Proc. Serono Symp. (Eds: Chin, W. W., Boime, I.), Norwell, MA, USA 1990 , 81.

[6] R.J. Ryan, M.C. Charlesworth, L.D. Erickson, D.J. McCormick, R.P. Milius, J.C. Morris III, in Glycoprotein Horm. Struct. Synth. Biol. Funct. Proc. Serono Symp. (Eds: Chin, W. W., Boime, I.), Norwell, MA, USA $1990,71$.

[7] S. Christakos, O.P. Bahl, J. Biol. Chem. 1979 ,254 , 4253.

[8] H. Sugino, G.R. Bousfield, W.T. Moore Jr, D.N. Ward, J. Biol. Chem. 1987, 262 , 8603.

[9] G.R. Bousfield, W.K. Liu, H. Sugino, D.N. Ward, J. Biol. Chem. 1987, 262 , 8610.

[10] G.R. Bousfield, V.Y. Butnev, V.Y. Butnev, Biol. Reprod.2001 , $64,136$.

[11] Y. Combarnous, F. Guillou, N. Martinat, Endocrinology1984 , 115 , 1821.

[12] H.H. Cole, M. Bigelow, J. Finkel, G.R. Rupp,Endocrinology 1967, 81 , 927. 
[13] B.B. Aggarwal, H. Papkoff, Domest. Anim. Endocrinol.1985 , 2 , 173.

[14] D. Schams, C. Mentzer, E. Schallenberger, J. Hahn, H. R., in Control Reprod. Cow, Sreenan J. Ed., The Hague: Martinus Nijhoff, Galway, Irland 1978, 122.

[15] S.D. Martinuk, A.W. Manning, W.D. Black, B.D. Murphy,Biol. Reprod. 1991 , $45,598$.

[16] F. De Rensis, F. Lopez-Gatius, Reprod. Domest. Anim.2014 , 49 , 177.

[17] H.D. McClamrock, Clin. Obstet. Gynecol. 2003 ,46, 298.

[18] S. Legardinier, C. Cahoreau, D. Klett, Y. Combarnous, Reprod. Nutr. Dev. 2005 , 45 , 255.

[19] S. Legardinier, M. Duonor-Cerutti, G. Devauchelle, Y. Combarnous, C. Cahoreau, J. Mol. Endocrinol. $2005,34,47$.

[20] A. Ubach, PhD. Thesis, Universitat Autonoma de Barcelona2009 .

[21] A.F. Ingles, PhD. Thesis , Universitat Autonoma de Barcelona 2012.

[22] Y. Durocher, M. Butler, Curr. Opin. Biotechnol.2009, 20, 700.

[23] A. Jablonka-Shariff, J.F. Roser, G.R. Bousfield, M.W. Wolfe, L.E. Sibley, M. Colgin, I. Boime, Theriogenology $2007,67,311$.

[24] M.J. Yoon, I. Boime, M. Colgin, K.D. Niswender, S.S. King, M. Alvarenga, A. Jablonka-Shariff, C.A. Pearl, J.F. Roser, Domest. Anim. Endocrinol. 2007 , 33 , 470.

[25] E. Harlow, D. Lane, in Antibodies. A Lab. Man., Cold Spring Harbor Laboratory Press, New York, USA 1988 , Ch. 5 .

[26] E.F. Mufarrege, S. Antuna, M. Etcheverrigaray, R. Kratje, C. Prieto, Protein Expr. Purif. 2013 , 95 , 50.

[27] T. Dull, R. Zufferey, M. Kelly, R.J. Mandel, M. Nguyen, D. Trono, L. Naldini, J. Virol. 1998 , 72 , 8463.

[28] H. Miyoshi, U. Blomer, M. Takahashi, F.H. Gage, I.M. Verma,J. Virol. 1998 , 72 , 8150.

[29] R. Freshney, in Cult. Anim. cells. A Man. basic Tech.,Wiley-Liss Inc, New Jersey, USA 2000, Ch. 9.

[30] J.P. Mather, Methods Enzymol. 1990 , 185 , 567.

[31] J.A. Cook, J.B. Mitchell, Anal. Biochem. 1989 ,179 , 1.

[32] U.K. Laemmli, Nature 1970, 227 , 680.

[33] B.D. Hames, in Gel Electrophor. proteins. A Pract. approach, (Eds: Hames, B. D., Rickwood, D.), Oxford University Press, Inc., New York, USA 1990 , Ch. 1.

[34] H.H. COLE, J. ERWAY, Endocrinology 1941,29 , 514.

[35] A.J. Edmonson, I.J. Lean, L.D. Weaver, T. Farver, G. Webster,J. Dairy Sci. 1989 , 72 , 68.

[36] M. Schneider, I.W. Marison, U. Von Stockar, J. Biotechnol. 1996 , 46 , 161.

[37] M. Bollati-Fogolin, G. Forno, M. Nimtz, H.S. Conradt, M. Etcheverrigaray, R. Kratje, Biotechnol. Prog. $2005,21,17$.

[38] M. Crowe, Reprod. Domest. Anim. 2008 ,43 , 20.

[39] R. Nunez-Olivera, T. De Castro, C. Garcia-Pintos, G. Bo, J. Piaggio, A. Menchaca, Anim. Reprod. Sci. $2014,146,111$. 
[40] A.H. Souza, S. Viechnieski, F.A. Lima, F.F. Silva, R. Araujo, G.A. Bo, M.C. Wiltbank, P.S. Baruselli, Theriogenology 2009 , 72, 10.

[41] I. Garcia-Ispierto, I. Lopez-Helguera, A. Martino, F. Lopez-Gatius, Reprod. Domest. Anim. 2012 , 47 , 752 .

[42] P. Lonergan, L. O’hara, N. Forde, Anim. Reprod.2013 , 10 , 223.

[43] M. Hesser, J. Morris, J. Gibbons, Reprod. Domest. Anim.2011 , 46 , 933.

Table 1. Effects of reCG or PMSG application at intravaginal device removal in an ovulation synchronization protocol $(\mathrm{N}=63)$.

\begin{tabular}{|c|c|c|c|c|}
\hline VARIABLE & CONTROL & reCG & PMSG & P-VALUE \\
\hline $\mathbf{N}$ & 22 & 20 & 21 & \\
\hline BCS & $2.71 \pm 0.2$ & $2.72 \pm 0.2$ & $2.71 \pm 0.16$ & 0,33 \\
\hline DFDd7 (mm) & $9.1 \pm 2.1$ & $9.3 \pm 2.1$ & $8.8 \pm 2.5$ & 0.744 \\
\hline DFDd9 (mm) & $9.94 \pm 2.1$ & $11.02 \pm 1.9$ & $10.02 \pm 2.4$ & 0.260 \\
\hline OR & $41 \%(9 / 22)^{b}$ & $80 \%(16 / 20)^{a}$ & $71 \%(16 / 21)^{\mathrm{a}}$ & 0.016 \\
\hline OT (h) & $57 \pm 3.6^{\mathrm{a}}$ & $63 \pm 2.8^{\mathrm{a}}$ & $73 \pm 3.5^{b}$ & 0.004 \\
\hline CLD (mm) & $18.2 \pm 2.7$ & $18.0 \pm 3.5$ & $15.9 \pm 4.2$ & 0.157 \\
\hline
\end{tabular}

a) Values with different superscript letters are significantly different $(\mathrm{P}<0.05)$

\section{FIGURE LEGENDS}

Fig. 1: Target species study experimental design . US $=$ ultrasound exam; $E B=$ estradiol benzoate; $\mathrm{EC}=$ estradiol cypionate; $\mathrm{P} 4=$ progesterone; $\mathrm{IVD}=$ intravaginal device.

Fig. 2: Culture of P5C3 clone in a $1 \mathrm{~L}$ bioreactor. Evolution of cell density, cell viability, lactate, glucose and reCG concentration along time. Temperature and perfusion rate per day are shown. Asterisk character indicates cell bleeding.

Fig. 3: SDS-PAGE of fractions corresponding to different stages of reCG purification. Samples were subjected to electrophoresis under non-reducing conditions and stained with Coomassie blue. Lane MW, Blue Plus@ Protein Marker (Transgene Biotech); lane 1, clarified supernatant; lane 2, flowthrough; lane 3, wash; lane 4, eluate.

Fig. 4: Western blot analysis of reCG under different conditions. A: Unheated samples were electrophoresed under non-reducing conditions. B: Heated samples were electrophoresed under non-reducing conditions. C: Heated samples were electrophoresed under reducing conditions. After SDS-PAGE proteins were probed with antiserum against reCG. For each panel, lane 1 corresponds to PMSG, lane 2 to reCG, lane 3 to supernatant of sCHOwt, and lane MW to ECL ${ }^{\mathrm{TM}}$ Rainbow ${ }^{\mathrm{TM}}$ Marker $\left(\right.$ Amersham $\left.^{\mathrm{TM}}\right)$.

Fig. 5: IEF profile of PMSG (lane 1) and reCG (lane 2) glycoforms. After separation, proteins were transferred onto PVDF membrane and probed with rabbit polyclonal sera anti-reCG. The line indicates the range of $\mathrm{pH}$ employed. 

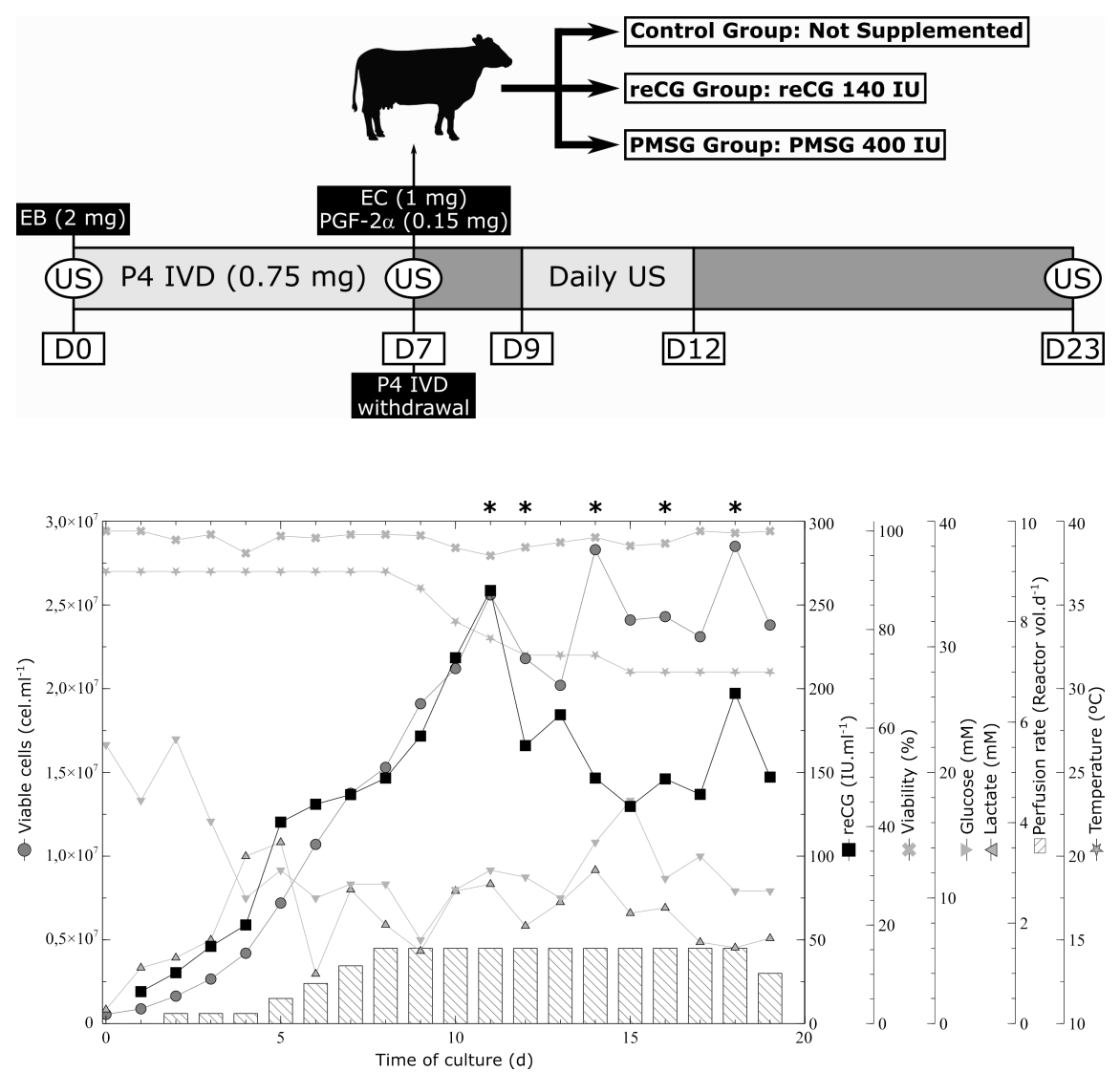

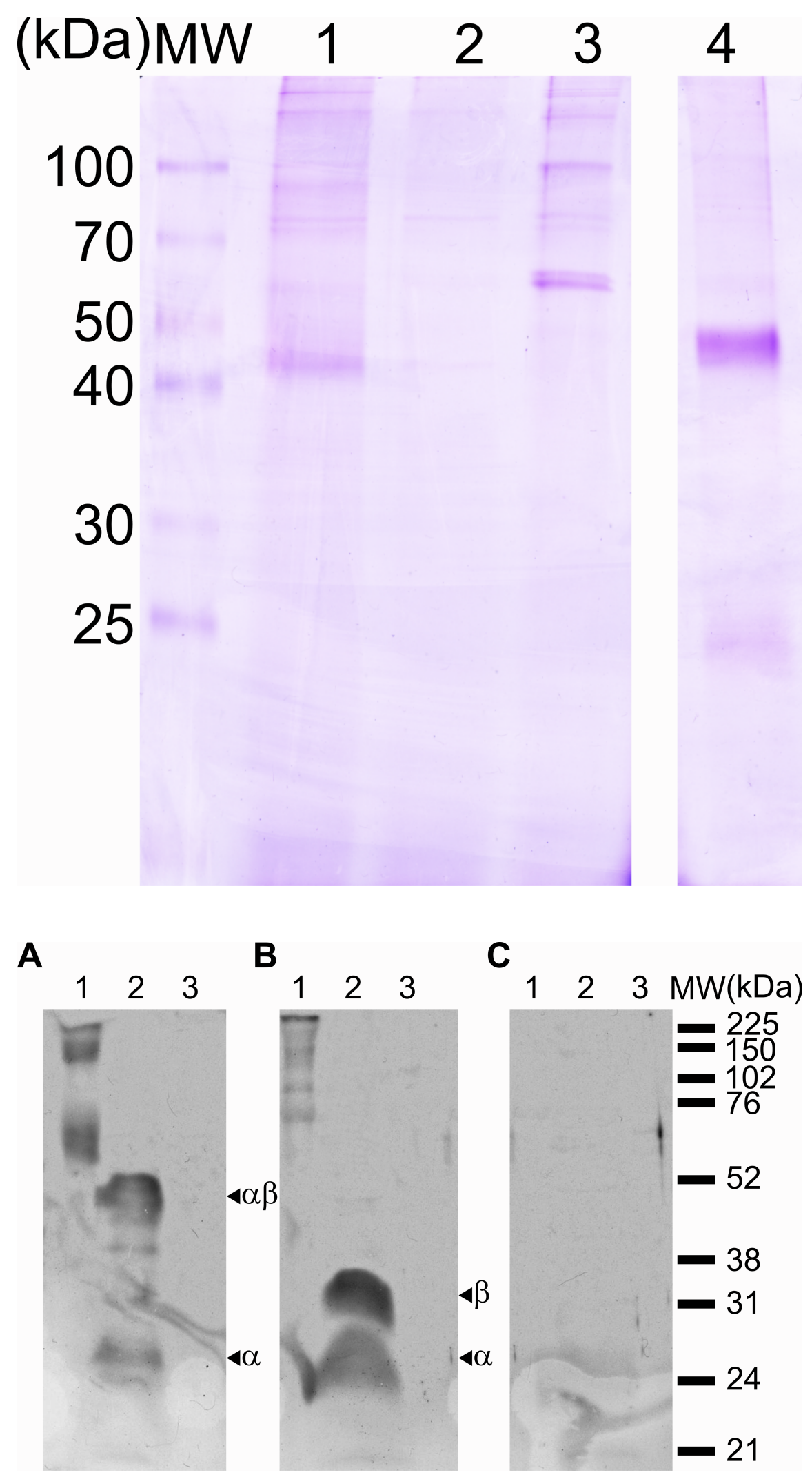


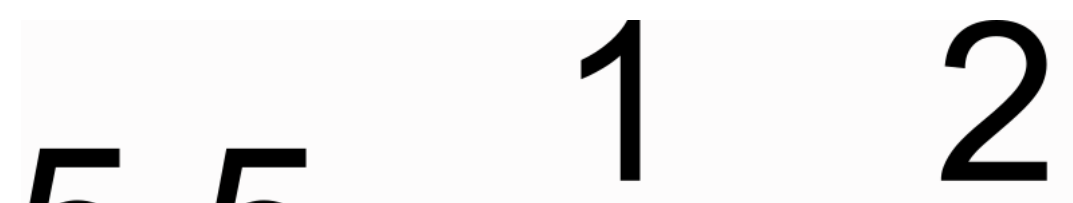

5,5

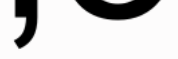

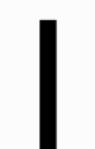

